\title{
Fourier series analysis of fractal lenses: theory and experiments with a liquid-crystal display
}

\author{
Jeffrey A. Davis, Sean P. Sigarlaki, Julia M. Craven, and María Luisa Calvo
}

\begin{abstract}
We report on a Fourier series approach that predicts the focal points and intensities produced by fractal zone plate lenses. This approach allows us to separate the effects of the fractal order from those of the lens aperture. We implement these fractal lenses onto a liquid-crystal display and show experimental verification of our theory. (C) 2006 Optical Society of America

OCIS codes: $\quad 050.1940,230.6120,230.3720,050.1970,080.3630$.
\end{abstract}

\section{Introduction}

Fractal lenses have been a topic of recent interest ${ }^{1-3}$ because the number of focal points increases as the growth stage of the fractal increases. We describe the fractal lens as the product of binary Fresnel lenses. These Fresnel lenses are analyzed by a Fourier series approach. By forming the fractal lens from the product of these Fresnel lenses, we can predict the positions and intensities of the fractal focal points. Moreover, we can separate the effects of the product of the lenses from the effects of the aperture for the entire lens function. This theoretical approach also allows us to explore the dimensionality of the fractal structure.

Because our approach is different from the previous theory of fractal lenses, ${ }^{1}$ we first review the focusing properties of phase lenses and Fresnel lenses. Then we apply these results to the fractal lens. Experimental results confirm our approach.

\section{Focusing Properties of Fractal Lenses}

\section{A. Fraunhofer Diffraction with a Converging Lens}

We assume a circularly symmetric transmission lens function $t\left(r_{1}\right)$, where $r_{1}$ represents the coordinate in the input plane. Using Fresnel diffraction and assuming axial symmetry yield the scalar electric field

J. A. Davis (jdavis@sciences.sdsu.edu), S. P. Sigarlaki, and J. M. Craven are with the Department of Physics, San Diego State University, San Diego, California 92182-1233. M. L. Calvo is with the Departamento de Optica, Facultad de Ciencias Fisicas, Universidad Complutense de Madrid, 28040 Madrid, Spain.

Received 24 May 2005; revised 29 August 2005; accepted 30 August 2005.

$0003-6935 / 06 / 061187-06 \$ 15.00 / 0$

(C) 2006 Optical Society of America at the origin of the viewing plane $\left(x_{2}=y_{2}=0\right)$ located a distance $z$ from the input plane:

$$
E\left(x_{2}=0, y_{2}=0\right)=\frac{1}{\lambda z} \int_{0}^{\infty} t\left(r_{1}\right) \exp \left(\frac{i k r_{1}^{2}}{2 z}\right) 2 \pi r_{1} \mathrm{~d} r_{1}
$$

We examine only the origin of the output plane because we are interested in the axial distribution of the energy diffracted by the fractal lens. We ignore constant phase terms and $k=2 \pi / \lambda$, where $\lambda$ is the wavelength.

We apply the approach of Ref. 1 such that we can generate expressions for binary Fresnel lenses and fractal lenses by using the same notation. We begin with a one-dimensional linear grating phase function, given by

$$
g(x)=\exp (-i 2 \pi x / d)
$$

This function is shown in Fig. 1(a), in which gray levels represent the phase. The grating has period $d$ and length $L$, and the origin of the system is at the left for convenience.

In designing a lens that has a known focal length $f$ from this pattern, one must scale the locations of periods $d_{n}$ of this grating nonlinearly, as shown in Fig. 1(b), to positions $r_{n}$ such that $r_{n}=\sqrt{2 n \lambda f}$. These values correspond to the points where the phase term in Eq. (2) changes by $2 \pi n$, where $n$ is an integer. Length $L$ of the grating is scaled to aperture $a$ of the lens. Finally, the scaled function is rotated about the axis of symmetry to yield the radially symmetric 
(a)

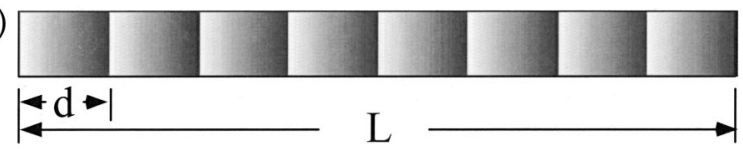

(b)

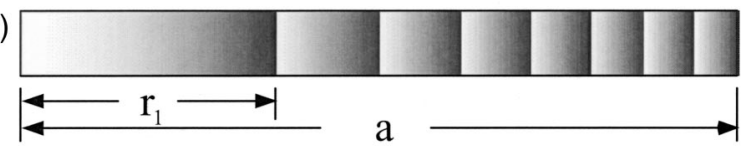

(c)

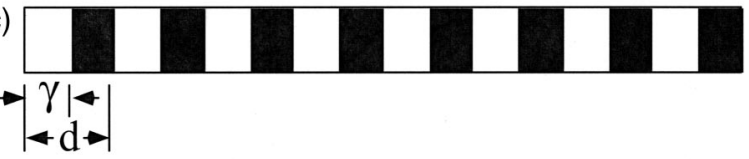

(d)

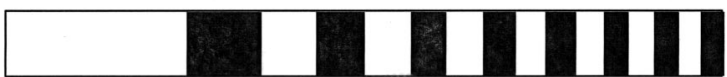

Fig. 1. Sequence showing (a) a one-dimensional linear phase function, (b) a one-dimensional lens phase pattern, (c) a binarized one-dimensional linear phase function, and (d) a binarized onedimensional lens pattern.

function for a converging lens with focal length $f$ as

$$
t\left(f, r_{1}\right)=\exp \left(\frac{-i k r_{1}^{2}}{2 f}\right)
$$

Using this lens transmission function, we rewrite the Fresnel diffraction in Eq. (1) as

$$
\begin{aligned}
E\left(x_{2}\right. & \left.=0, y_{2}=0\right) \\
& =\frac{1}{\lambda z} \int_{0}^{a} \exp \left(\frac{-i k r_{1}^{2}}{2 f}\right) \exp \left(\frac{i k r_{1}^{2}}{2 z}\right) 2 \pi r_{1} \mathrm{~d} r_{1} .
\end{aligned}
$$

This integral yields a focused spot located at distance $z=f$ from the input plane.

The authors of Ref. 1, however, show a more useful derivation. First, they define a normalized axial coordinate $u=a^{2} / 2 \lambda z$, and we define a new axial coordinate when $z=f$ as $u_{0}=a^{2} / 2 \lambda f$. Consequently the axial coordinates increase as aperture $a$ increases.

Next, they ${ }^{1}$ define a new variable $s$ as $s=r_{1}{ }^{2} / a^{2}$ - 0.5. With this change in variable, parameter $s$ varies from -0.5 to +0.5 , and the integral maintains its symmetry with respect to the new origin as

$$
\begin{aligned}
E\left(x_{2}\right. & \left.=0, y_{2}=0\right) \\
& =2 \pi u \int_{-0.5}^{0.5} \exp \left(-2 \pi u_{0} s\right) \exp (i 2 \pi u s) \mathrm{d} s .
\end{aligned}
$$

We can rewrite this integral as a Fourier-transform integral by introducing a rectangle function of unit support and extending the limits of integration to \pm infinity, as

$$
E(u)=2 \pi u \int_{-\infty}^{\infty} \operatorname{rect}(s) \exp \left(-i 2 \pi u_{0} s\right) \exp (i 2 \pi u s) \mathrm{d} s .
$$

Now the lens function can have an infinite aperture, while the rect function effectively limits the integral. We use the fact that the Fourier transform of the product of two functions is the convolution of their Fourier transforms and rewrite the integral of Eq. (6) as

$E(u)=2 \pi u \operatorname{sinc}(u) \otimes \delta\left(u-u_{0}\right)=2 \pi u \operatorname{sinc}\left[\left(u-u_{0}\right)\right]$.

Here the symbol $\otimes$ represents the convolution operation. The electric field strength varies as a sinc function in the $u$ direction. The center location of the sinc function is at $u_{0}$ and corresponds to the focal point of the lens. As lens aperture $a$ decreases, the focus broadens along the $z$ axis. Alternatively, when aperture $a$ approaches infinity, the sinc function is reduced to a delta function along the axial distance.

However, Eq. (7) predicts that the electric-field amplitude will decrease when we use a lens with a longer focal length because of the factor $u$ that multiplies this expression. This conclusion is misleading because it suggests that the focused energy increases as the focal length decreases, and this would violate the law of conservation of energy. The explanation for this conflict is subtle. What it really means is that the beam energy is spreading out in the radial direction. Consequently, to conserve energy, the maximum of the electric field amplitude at the origin must decrease. In practice the detector integrates the focused intensity over the area of the detector element; so, for clarity, we omit this initial multiplicative factor of $u$ for the remainder of this paper, or, equivalently, we normalize it as $2 \pi u=1$.

\section{B. Application to Binary Fresnel Zone Plates}

Now we extend the discussion to binary Fresnel zone plates. First we define a threshold for the phase in Fig. 1(a). All points above and below this threshold are assigned values of +1 and 0 , respectively. By adjusting the threshold level we can change width $\gamma$ for the +1 region and width $\varepsilon=d-\gamma$ for the 0 region, following the notation of Monsoriu et al. ${ }^{4}$ Consequently we obtain a binary grating as shown in Fig. 1(c).

Through the introduction of the rectangle function in Eq. (6), we can treat the gratings as having infinite length. Therefore we can rewrite the infinitely long binary diffraction grating in Fig. 1(c) as a Fourier series:

$$
t(x)=\sum_{l=-\infty}^{\infty} c_{l} \exp \left(\frac{-i l 2 \pi x}{d}\right) .
$$

Here the coefficients $c_{l}$ depend on the ratio $\gamma / d$ and the value of $l$ as

$$
c_{l}=\left(\frac{\gamma}{d}\right) \operatorname{sinc}\left(\frac{l \pi \gamma}{d}\right)
$$

Next we apply the stretching algorithm to obtain 


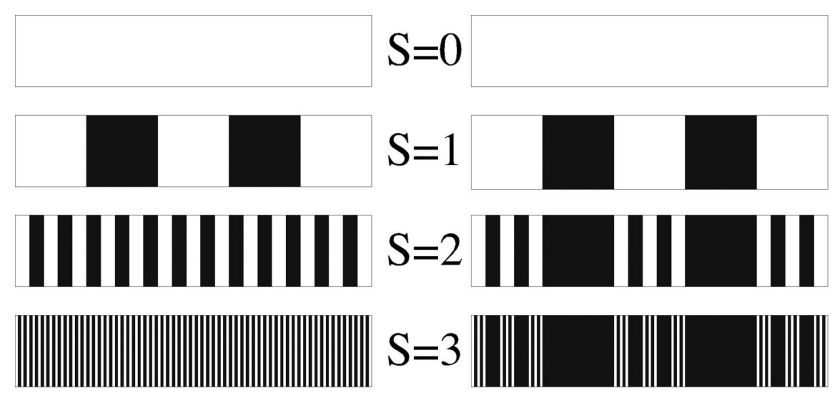

(a)

(b)

Fig. 2. Sequence showing generation of (a) Fresnel zone plates for $N=3$ and several values of $S$ and (b) fractal zone plates for $N=3$ and several values of $S$. Transparent and opaque segments are represented by white and black, respectively.

the scaled pattern in Fig. 1(d). After rotating this pattern about the axis of symmetry as before, we obtain a Fourier series of lenses as

$$
t\left(f, r_{1}\right)=\sum_{l=-\infty}^{\infty} c_{l} \exp \left(\frac{-i l k r_{1}^{2}}{2 f}\right)
$$

This infinite series of lens functions has both positive and negative focal lengths $f / l$ whose strengths are given by coefficients $c_{l}$.

The Fraunhofer diffraction from these lenses (we use the same approach as above) is given by

$$
E(u) \approx \sum_{l=-\infty}^{\infty} c_{l} \operatorname{sinc}\left[\left(u-l u_{0}\right)\right] .
$$

The Fresnel lens produces a series of focal points at locations of $l u_{0}$ that have intensities proportional to $c_{l} c_{l}{ }^{*}$. These focal points correspond to positions $z=f, z=f / 2, z=f / 3$, etc.

In the usual case in which $\gamma / d=1 / 2$, the coefficients are given by $c_{0}=1 / 2, c_{ \pm 1}=1 / \pi, c_{ \pm 2}=0$, $c_{ \pm 3}=1 / 3 \pi$, etc. Because all the even coefficients vanish, the intensities at all the even focal points are zero. If the ratio of $\gamma / d$ varies, the strengths of the focal points are changed. For example, if $\gamma / d=1 / 3$, then $c_{ \pm 3}=0$, while $c_{ \pm 2}=0.138$.

The advantages of this formalism are apparent. Fundamental focal length $f$ is related to the original period of the generating phase function, whereas the depth of focus is dependent only on aperture size $a$. As we now increase aperture $a$, each of the focus spots will get sharper along the $z$ direction. Next we apply this formalism to the generation of fractal lenses.

\section{Fractal Zone Plates}

We follow the approach in Ref. 1 to compare the generation of Fresnel and fractal lenses. Figure 2(a) shows a sequence for formulating Fresnel lenses with decreasing periods. We begin with an initiator bar of unit length $L$ and designated $S=0$, where $S$ is the stage. For the $S=1$ stage we divide this initial region into $N$ regions that have value 1 and into $N-1$ regions that have value 0 , where $N$ is defined as the order of the pattern. Note that the order can also be defined by the total number of segments as $2 N-1$. As before, the widths of the +1 regions are designated $\gamma$, while the widths of the 0 regions are designated $\varepsilon$. For progressive stages of $S$ we simply divide the previous region into $2 N-1$ segments. Each new segment is then divided into $N$ regions having a value of 1 and $N-1$ regions having a value of 0 . Figure 2(a) shows the stages for $S=0-3$ where the widths of the two regions are equal and $N=3$.

With this approach, overall length $L$ is constant. The periods of the gratings decrease as $S$ increases. When the stretching algorithm is applied and the subsequent scaled function is rotated, we obtain a series of Fresnel lenses for which the primary focal lengths will decrease from top to bottom.

Figure 2(b) shows the generation of fractal gratings for $S=0-3$ where $N=3$. The only difference here from Fig. 2(a) is that, as we go from one stage to the next, the areas that have values of zero retain this value. Again, we apply the stretching algorithm and rotate the function to obtain the series of fractal lenses.

Next we introduce the most important point of this paper. It is obvious from comparing the two figures that we obtain the fractal gratings in Fig. 2(b) by multiplying the patterns in Fig. 2(a). This was the basis for our previous study, ${ }^{2}$ in which a fractal lens was formed by the product of several lenses. To form the final aperture, we simply use the central area of the next lens in the series. For example, the $S=3$ is formed from the product of lenses with focal lengths $f,(2 N$ $-1) f$, and $(2 N-1)^{2} f$. Finally, we multiply this product by the central aperture of a lens with focal length $(2 N-1)^{3} f$.

With this approach we always keep the same primary focal length $f$ for the fractal lens. As we increase the fractal order, we not only increase the number of lenses in the product but also increase the aperture of the lens. As we now recognize that the focal structure of the lens is independent of the aperture, the only effect of the aperture is to spread the focus along the axial direction.

This procedure solves many problems for the experimental implementation of these fractal lenses. It allows us to construct lenses of a desired focal length and to compare different fractal structures that have the same primary focal length. More importantly, this formalism allows us to analyze easily the focal properties of the fractal lenses.

To illustrate, we examine the case when $S=2$, where the transmission function is written as the product of a Fresnel lens with focal length $f$ and another Fresnel lens with focal length $(2 N-1) f$. The fractal lens is now written as the product of two Fourier series as

$$
\begin{aligned}
t\left(f, N, S=2, r_{1}\right)= & \sum_{l=-\infty}^{\infty} c_{l} \exp \left(\frac{-i l k r_{1}^{2}}{2 f}\right) \\
& \times \sum_{m=-\infty}^{\infty} \exp \left[\frac{-i m k r_{1}^{2}}{2(2 N-1) f}\right]
\end{aligned}
$$


We can combine terms as

$$
\begin{aligned}
& c_{l} c_{m} \exp \left(\frac{-i l k r_{1}^{2}}{2 f}\right) \exp \left[\frac{-i m k r_{1}^{2}}{2(2 N-1) f}\right] \\
&=c_{l} c_{m} \exp \left(\frac{-i k r_{1}^{2}}{2 f_{l m}}\right) .
\end{aligned}
$$

Each of these terms forms a different focus that has an electric field amplitude proportional to $c_{l} c_{m}$ and an effective focal length $f_{l m}$ given by

$$
\frac{1}{f_{l m}}=\stackrel{l}{f}+\frac{m}{(2 N-1) f} .
$$

The Fourier coefficients are given by $c_{0}=1 / 2, c_{ \pm 1}$ $=1 / \pi, c_{ \pm 2}=0, c_{ \pm 3}=1 / 3 \pi$, etc. Note that the coefficients vanish when either $l$ or $m$ is even.

Again we redefine our variables and convolve the structure with the aperture function. The output from the fractal lens is now written as

$$
E(u) \approx \sum_{l=-\infty}^{\infty} \sum_{m=-\infty}^{\infty} c_{l} c_{m} \operatorname{sinc}\left[\left(u-u_{l m}\right)\right] .
$$

Now we see the effects of the various parameters that govern the fractal lens. The order $N$ of the fractal determines the focal lengths of the lenses that are multiplied, while growth stage $S$ determines the number of lenses that are multiplied and the final number of focal points. However, these focal points are convolved with the sinc function from the aperture function. If the focal points are too close together, they might not be resolved. In our experiments, we deliberately increased the aperture function to sharpen the peaks in the axial direction.

The intensities associated with each focus depend on the coefficients $c_{l} c_{m}$ as

$$
I_{l m} \approx c_{l}^{2} c_{m}^{2} .
$$

However, several values of $l$ and $m$ might yield focus spots at the same location. In this case we first add all the coefficients $c_{l} c_{m}$ and then multiply the sum by its complex conjugate to obtain the final intensity. We shall return to this point later.

We can extend this formalism to create the $S=3$ fractal lens. Our product in Eq. (12) now contains a third product, with focal length $(2 N-1)^{2} f$, as

$$
\begin{aligned}
t(f, N, S= & \left.3, r_{1}\right)=\sum_{l=-\infty}^{\infty} c_{l} \exp \left(\frac{-i l k r_{1}^{2}}{2 f}\right) \\
& \times \sum_{m=-\infty}^{\infty} c_{m} \exp \left[\frac{-i m k r_{1}^{2}}{2(2 N-1) f}\right] \\
& \times \sum_{n=-\infty}^{\infty} c_{n} \exp \left[\frac{-i n k r_{1}^{2}}{2(2 N-1)^{2} f}\right] .
\end{aligned}
$$

As before, we can combine terms as

$$
\begin{aligned}
& c_{l} c_{m} c_{n} \exp \left(\frac{-i l k r_{1}{ }^{2}}{2 f}\right) \exp \left[\frac{-i m k r_{1}{ }^{2}}{2(2 N-1) f}\right] \\
& \times \exp \left[\frac{-i n k r_{1}{ }^{2}}{2(2 N-1)^{2} f}\right]=c_{l} c_{m} c_{n} \exp \left(\frac{-i l k r_{1}{ }^{2}}{2 f_{l m n}}\right) .
\end{aligned}
$$

Each of these terms will form a different focus with strength $c_{l} c_{m} c_{n}$ and an effective focal length $f_{l m n}$ given by

$$
\frac{1}{f_{l m n}}=\frac{l}{f}+\frac{m}{(2 N-1) f}+\frac{n}{(2 N-1)^{2} f} .
$$

The analysis of Eq. (17) shows the basic recurrent procedure for the fractal lenses. As growth stage $S$ of the fractal increases, the previous product of lenses is multiplied by another lens with a longer focal length. This causes splitting of each of the previous focal spots. Our new approach allows the focal lengths and relative intensities to be determined easily.

\section{Changing the Dimensionality of the Fractal Lens}

There are several variations on this recipe for forming fractal structures, as discussed in the literature. ${ }^{3-6}$ These variations depend primarily on the relative sizes of gaps $\varepsilon$ compared with the sizes of transparent areas $\gamma$. In Subsection 2.C we examined regular fractals, where $\gamma / d=1 / 2$.

In the first variation, the sizes of the gaps are still equal to each other, but they are no longer equal to the transparent areas. This is referred to as the dimensionality $D$ of the fractal and is defined as

$$
D=\frac{\ln N}{\ln (1 / \gamma)} .
$$

This extension is easily handled with our theoretical framework. The previous analysis would be exactly the same. However, we saw from Eq. (9) that the strengths of Fourier coefficients $c_{l}$ are given by the ratio $\gamma / d$. Consequently the effect of changing the dimensionality is merely to change the strengths of the various focal points. For example, when we chose $\gamma / d=1 / 2$, all the even coefficients vanished. However, if we instead choose $\gamma / d=1 / 3$, then, for example, coefficient $c_{3}$ would vanish. Therefore we can control the strengths of the various focal spots by changing the dimensionality. We shall show experimental evidence for this.

We note for completeness that there have been some recent studies of fractal structures with varying lacunarity. ${ }^{3-6}$ The lacunarity of the fractal structure is changed when the widths of the gaps are not constant (as they were in Fig. 2) but vary with position. These structures are more difficult to analyze with the Fourier-transform approach. However our formalism easily handles changes in the dimensionality.

\section{Experimental Results}

Experimental results were obtained with a parallelaligned LCD manufactured by Seiko Epson, ${ }^{7}$ with 


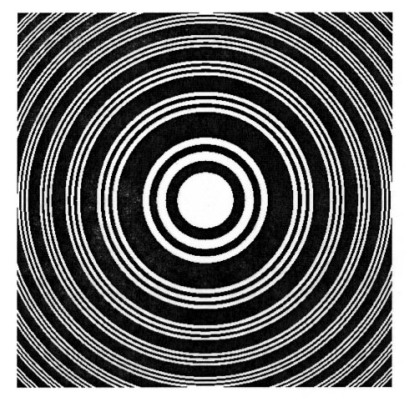

(a)

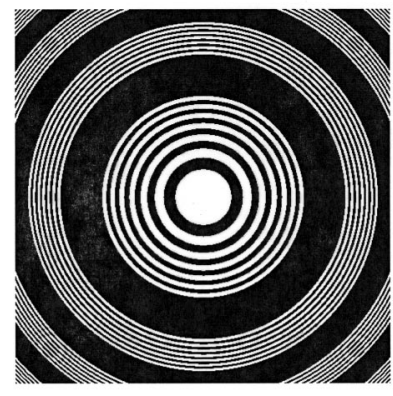

(c)

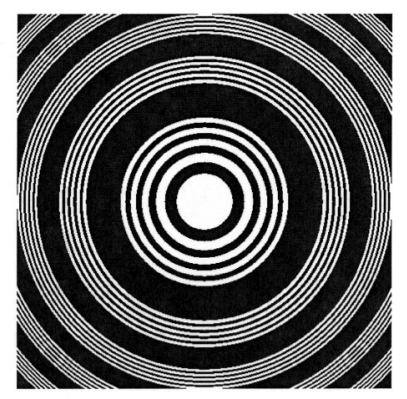

(b)

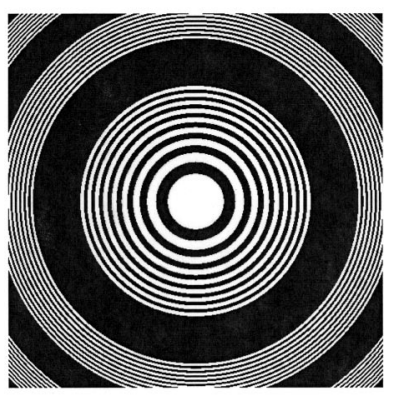

(d)
Fig. 3. Fractal lens patterns for $S=2$ and $N=$ (a) 3, (b) 5, (c) 7, (d) 9 .

$640 \times 480$ pixels on a 1.3 in. $(3.3 \mathrm{~cm})$ diagonal display. The pixel spacing was $42 \mu \mathrm{m}$. We operated the display in a binary phase-only mode. The LCD was illuminated with collimated light at $514 \mathrm{~nm}$ from an argon laser and focused by the fractal lens encoded onto the LCD. The focused spots were detected with a DataRay CCD camera as a function of distance from the LCD.

Some fractal lens patterns are shown in Fig. 3 for $N=3,5,7,9$ and $S=2$. Here we use a large focal length of $5 \mathrm{~m}$ to see more clearly the structure near the center of the lens. For example, we form the $N=7$ lens by multiplying the Fresnel lens pattern for the $5 \mathrm{~m}$ lens by the Fresnel lens pattern for a lens with focal length of $(2 N-1) f$ or $65 \mathrm{~m}$.

For our experiments we used values of $N=2-9$ and kept the parameter $S=2$. In all cases our aperture size was the full size of the spatial light modulator and the fundamental focal length was $1.5 \mathrm{~m}$. Experimental results are shown in Fig. 4 for the locations of the three transverse focal points, $f_{1,0}, f_{1,1}$, and $f_{1,-1}$, for $N=2-9$ and $S=2$. These results agree extremely well with the theoretical points from Eq. (14) that are designated by the lines. Note that the lines are guides for the eye only.

We also examined other focal points and their intensities. Table 1 shows the locations and intensities for the series of lenses where $l=1$ and where $m$ ranges from -5 to 5 for $N=7$ and $S=2$. We selected this example because the focal points were quite close and we did not have significant overlap from other

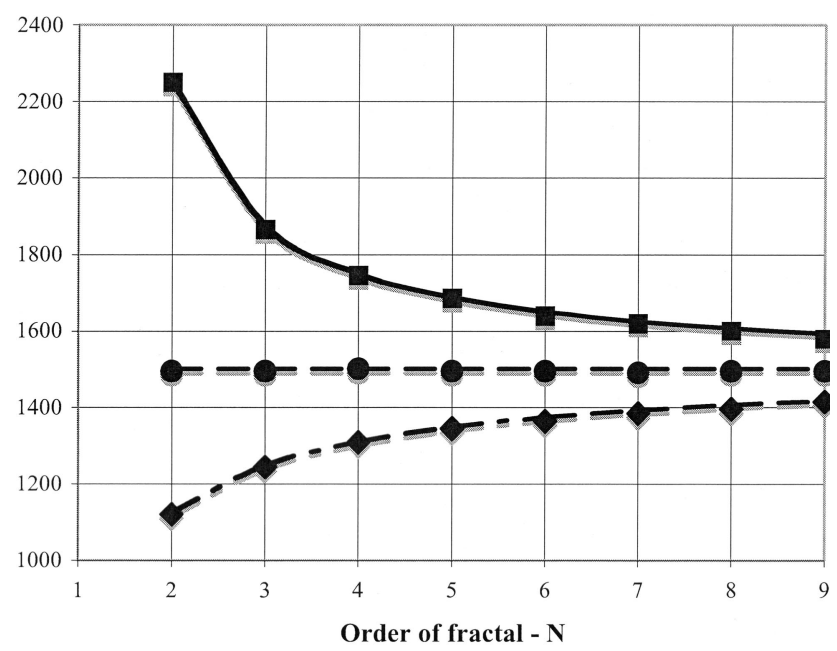

Fig. 4. Measured and theoretical positions of primary focal points corresponding to $f_{1,-1}$ (squares), $f_{1,0}$ (circles), and $f_{1,1}$ (diamonds) for $N=2-9$ and $S=2$.

values of $l$. For both theory and experiments, the values are normalized relative to the central and strongest focus at $f_{1,0}$. Errors in the intensity are a few percent and are due primarily to fluctuations in the laser intensity and the background intensity. Note that there were no focus spots corresponding to even values of $m$, again in agreement with theory. We could not measure the focal point for $m=-5$ because our optical bench is not long enough.

The intensities should follow the products $c_{l}{ }^{2} c_{m}{ }^{2}$ as covered in Eq. (16). For example, the ratios of the intensities at the focus spots $f_{1,0}, f_{1,1}$, and $f_{1,-1}$ should follow the relationship

$$
\frac{I_{1,0}}{I_{1, \pm 1}}=\frac{c_{1}^{2} c_{0}^{2}}{c_{1}^{2} c_{1}^{2}}=\frac{\pi^{2}}{4} \approx 2.5
$$

Consequently the main focus at $f_{1,0}$ should be 2.5 times more intense than either of the two subsidiary focal points, in good agreement with the experimental results.

Table 1. Experimental Data for Intensities and Focal Point Locations Corresponding to a Fractal Lens for $N=7$ and $S=2$

\begin{tabular}{ccccccc}
\hline & & \multicolumn{2}{c}{ Focus (mm) } & & \multicolumn{2}{c}{ Intensity (\%) } \\
\cline { 6 - 7 }$l$ & $m$ & Theory & Exp. & & Theory & Exp. \\
\hline 1 & 5 & 1083 & 1072 & & 1.6 & 4.2 \\
1 & 4 & 1147 & & & 0 & 0 \\
1 & 3 & 1219 & 1215 & & 4.5 & 5.6 \\
1 & 2 & 1300 & & & 0 \\
1 & 1 & 1393 & 1385 & & 40 & 34 \\
1 & 0 & 1500 & 1500 & & 100 & 100 \\
1 & -1 & 1620 & 1625 & & 40 & 36 \\
1 & -2 & 1773 & & & 0 \\
1 & -3 & 1950 & 1945 & & 4.5 & 5.4 \\
1 & -4 & 2167 & & & 0 & \\
1 & -5 & 2437 & & & 1.6 & \\
\hline
\end{tabular}


We did note that, for small values of $N$, the intensity of the farther focus at $f_{1,-1}$ for $l=1, m=-1$ was stronger than the intensity of the closer focus at $f_{1,1}$ for $l=1, m=1$. Our initial theory predicted that these intensities should be equal. However, different values for $l$ and $m$ can produce focus spots at the same location. The electric field amplitudes from these terms must be added and then the intensity determined.

For the farthest focal point, identical focus points are given by $f_{1,-1}$ and $f_{-1,4 N-3}$. For the closest focal point, identical focus points are given for $f_{1,1}$ and $f_{-1,4 N-1}$. The size of the farthest contribution from the term $l=-1, m=4 N-3$ is greater than the size of the closest contribution from the terms $l=-1$, $m=4 N-1$. Consequently the total intensity for the two contributions $(l=1, m=-1$ and $l=-1$, $m=4 N-3)$ at the farthest focal point will be stronger than the total intensity for the two contributions $(l=1, m=1$ and $l=-1, m=4 N-1)$ at the closest focal point. This difference in intensities will decrease as the value of $N$ increases because the sizes decrease with larger values of $l$ or $m$ in Eq. (9). Although our data support this theory, our experimental error is not sufficient to prove this conclusively.

In all lenses treated above, we kept the parameter $\gamma / d=1 / 2$. Finally, we changed the dimensionality of the second lens function [with focal length $(2 N$ - 1)f] such that $\gamma / d=1 / 3$. For this case, the focus spots at 1219 and $1950 \mathrm{~mm}$ corresponding to $\mathrm{m}=$ \pm 3 disappeared while the focus spots at 1300 and $1773 \mathrm{~mm}$ corresponding to $m= \pm 2$ appeared in agreement with theory.

\section{Conclusions}

We have introduced a Fourier analysis for fractal lenses that predicts the strengths and locations of the focal points for fractal lenses. We tested this theory for various fractal lenses that were encoded onto a programmable liquid-crystal display. Experimental results are in good agreement with this theory.

We thank Tomio Sonehara of Seiko Epson Corporation for the use of the LCD and Don Cottrell for writing the computer program.

\section{References}

1. G. Saavedra, W. D. Furlan, and J. A. Monsoriu, "Fractal zone plates," Opt. Lett. 28, 971-973 (2003).

2. J. A. Davis, L. Ramirez, J. A. R. Martin-Romo, T. Alieva, and M. L. Calvo, "Focusing properties of fractal zone plates: experimental implementation with a liquid-crystal display," Opt. Lett. 29, 1321-1323 (2004).

3. L. Zunino and M. Garavaglia, "Frauhofer diffraction by Cantor fractals with variable lacunarity," J. Mod. Opt. 50, 717-728 (2003).

4. J. A. Monsoriu, G. Saavedra, and W. D. Furlan, "Fractal zone plates with variable lacunarity," Opt. Express 12, 4227-4234 (2004).

5. D. L. Jaggard and A. D. Jaggard, "Polyadic Cantor superlattices with variable lacunarity,” Opt. Lett. 22, 145-147 (1997).

6. A. D. Jaggard and D. L. Jaggard, "Scattering from fractal superlattices with variable lacunarity," J. Opt. Soc. Am. A 15, 1626-1635 (1998).

7. J. A. Davis, P. Tsai, D. M. Cottrell, T. Sonehara, and J. Amako, "Transmission variations in liquid crystal spatial light modulators caused by interference and diffraction effects," Opt. Eng. 38, 1051-1057 (1999). 